\title{
0

\section{Occupational Exposure to Microbial Volatile Organic Compounds in a Waste-handling Unit}

Viegas, S. 1,2; Malta-Vacas, Joana1; Viegas, C. ${ }^{1}$

${ }^{1}$ Escola Superior de Tecnologia da Saúde de Lisboa - IPL; ${ }^{2}$ CIESP - Centro de Investigação e Estudos em Saúde Pública, Escola Nacional de Saúde Pública, ENSP, Universidade Nova de Lisboa

\section{Introduction}

Airborne fungal contaminants in waste-handling facilities are widely discussed in regard to health hazards for workers. Apart from pathogenicity, the potential impact of bioaerosols on health is being widely discussed from both allergenic and toxicological points of view. In this occupational setting, VOCs presence can be due to some compounds produced by fungi that are volatile and are released directly into the air. These are known as microbial volatile organic compounds (MVOCs) and are produced in the metabolism of microorganisms. MVOCs are formed during both the primary metabolism and the secondary metabolism as side-products, mainly in the metabolic oxidation of glucose from various intermediates. ${ }^{1}$

The production of MVOCs by fungi has been taken into account especially from the viewpoint of indoor pollution with microorganisms but the relevance of fungal metabolites in working environments has not been sufficiently studied.

\section{Aim of the Study}

\section{Determination of MVOCs presence in a waste-handling} unit.

\section{Materials and methods}

$\checkmark$ Multirae equipment (RAE Systems) was used to measured MVOCs concentration with a $10.6 \mathrm{eV}$ lamp.

$\checkmark$ The measurements were done near the workers breathing zone and during the normal activities developed.

$\checkmark$ All measurements were done continuously and had the duration of $5 \mathrm{~min}$. The higher value obtained in each measurement was considered.

$\checkmark$ In addition, for knowing fungi contamination, five air samples of 50 litres were collected through impaction method at 140 L/minute, at one meter height, onto malt extract agar with chloramphenicol (MEA).
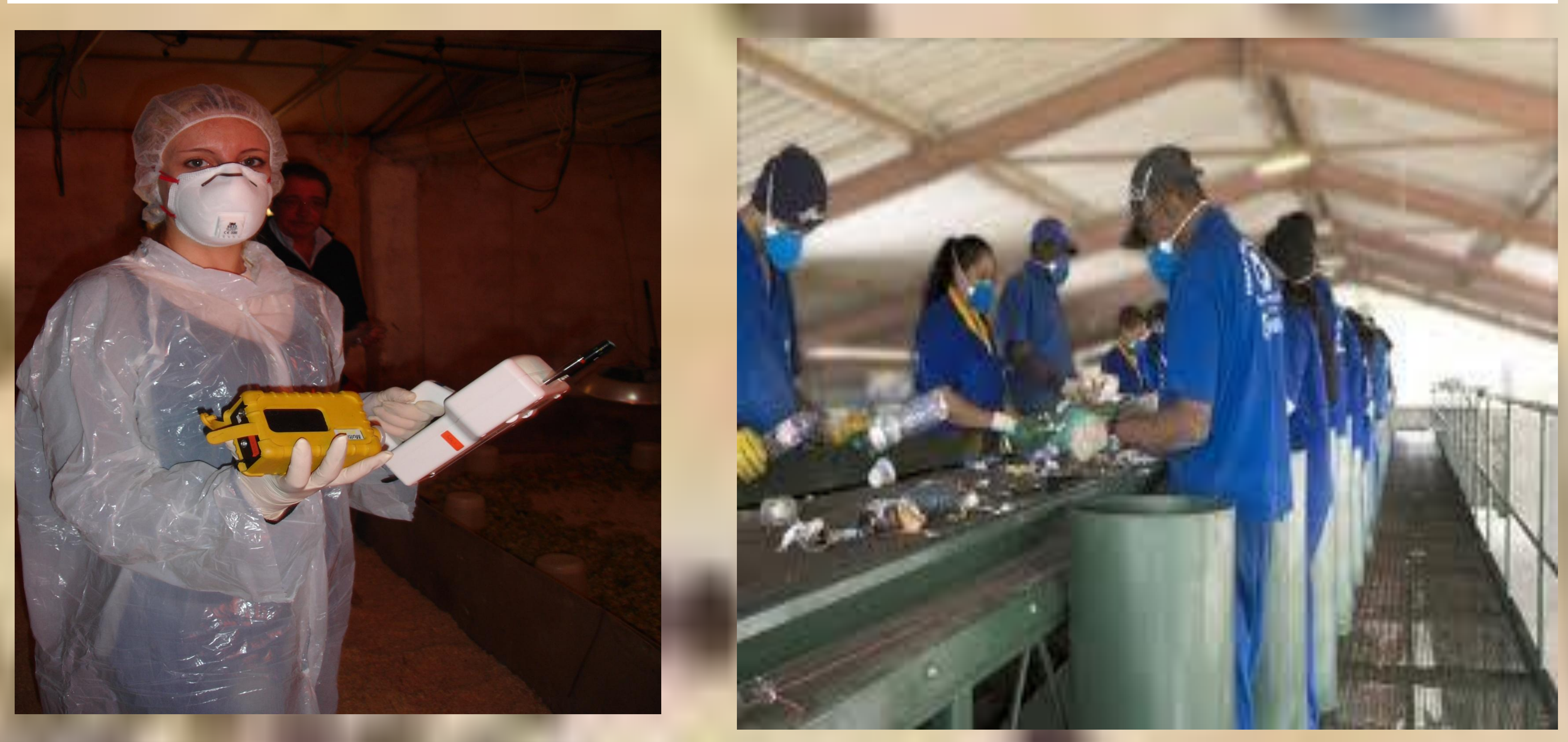

Fig. 1 and 2 Exposure assessment in waste-handling unit

\section{Results and Discussion}

\section{MVOCs results}

$\checkmark$ Range between $4.7 \mathrm{ppm}$ and $8.9 \mathrm{ppm}$ in the 6 locations considered.

Results are almost five times higher than normally obtained in indoor settings.

\section{Fungi results}

Two genera were identified in air: genus Penicillium found in all the samples in uncountable colonies and Rhizopus only in one sample (40 UFC/m³).

These fungi are known as MVOCs producers, namely terpenoids, ketones, alcohols and others.

Considering Penicillium species, themost common genus found, there are some related MVOCS described, in particular terpenes, specifically iso-longifolene, (+)-thujopsene, 10-epi$\beta$-acoradiene, $\beta$-chamigrene and widdra-2,4-diene. 2,3

Although in our study was not possible to determine the precise MVOCs present, it's important to take into consideration that these substances are significant indicators for air pollution caused by fungi. ${ }^{1}$

\section{Conclusions}

$\checkmark$ This occupational setting presents a mixture of biological and chemical exposures, which difficult health risk assessment.

$\checkmark$ Further research should concentrate on quantitative analyses of specific MVOCs to estimate potential health effects.

$\checkmark$ The evidence of possible negative health effects related with these exposures suggests a need of preventive and protective measures.

\section{References}

1. Korpi, A., Järnberg, J. \& Pasanen,A. (2006). 138. Microbial volatile organic compounds (MVOCs). National Institute for Working life. The Nordic Expert Group for Criteria Documentation of Health Risks from Chemicals. ISBN 978-91-7045-815-6.

2. Karlshøj, K. \& Larsen, T.O. (2005). Differentiation of species from the Penicillium roqueforti group by volatile metabolite profiling. Journal of Agricultural and Food Chemistry, $53,708-715$.

3. Jeleń, H. (2002). Volatile sesquiterpene hydrocarbons characteristic for Penicillium roqueforti strains producing PR toxin. Journal of Agricultural and Food Chemistry, 50, (22), 6569-6574. 